\title{
Asociación de parasitismo gastrointestinal con parámetros fisio- lógicos en ovinos mejorados de la Región Junín, Perú
}

\author{
Association of gastrointestinal parasitism with physiological parameters in \\ improved sheep of Junín Region, Peru
Fernando Arauco Villar ${ }^{1,5}$, Ide Unchupaico Payano², Noemí Mayorga Sánchez ${ }^{3}$, Danny Cruz Flores ${ }^{4}$

\section{Resumen}

\begin{abstract}
Se determinó la asociación del parasitismo gastrointestinal con el peso vivo, hematocrito y el método FAMACHA en ovinos de raza Junín (borreguillas y carnerillos) del lote Stud de la SAIS Túpac Amaru Ltda. N 1, Región Junín, Perú. El estudio se realizó en dos periodos estacionales: mayo (sequía) y octubre (lluvias). Se muestrearon 109 carnerillos y 98 borreguillas en mayo y a 89 carnerillos y 85 borreguillas en octubre de 2018. El peso de las hembras fue $39.75 \pm 0.21$ y el de machos de $43.31 \pm 0.20$, con diferencia significativa en la interacción sexo y mes $(\mathrm{p}<0.05)$, así como el valor del hematocrito entre meses $(p<0.05)$. Hubo correlación negativa entre el hematocrito y el valor transformado de carga parasitaria. Todos los animales estuvieron parasitados, encontrando una mayor carga parasitaria en machos en octubre y en mayo en las hembras. En mayo se presentaron más casos de animales resistentes al parasitismo que en octubre. La mayor frecuencia de parásitos gastrointestinales fue de Nematodirus (50\%), seguido de Trichostrongylus $(25 \%)$ y de Ostertagia $(15 \%)$.
\end{abstract}

Palabras clave: FAMACHA, hematocrito, nematodos gastrointestinales, peso vivo, carnerillos, borreguillas

\footnotetext{
${ }^{1}$ Facultad de Zootecnia, Universidad Nacional del Centro del Perú, Huancayo, Perú

${ }^{2}$ Universidad Nacional Intercultural de la Selva Central Juan Santos Atahualpa, Junín, Perú

${ }^{3}$ Laboratorio de Microbiología, Universidad Nacional del Centro del Perú, Huancayo, Perú

${ }^{4}$ Consultor Privado, Huancayo, Perú

${ }^{5}$ E-mail:faraucov@gmail.com
}

Recibido: 17 de agosto de 2019

Aceptado para publicación: 23 de octubre de 2021

Publicado: 22 de diciembre de 2021

CLos autores. Este artículo es publicado por la Rev Inv Vet Perú de la Facultad de Medicina Veterinaria, Universidad Nacional Mayor de San Marcos. Este es un artículo de acceso abierto, distribuido bajo los términos de la licencia Creative Commons Atribución 4.0 Internacional (CC BY 4.0) [https:// creativecommons.org/licenses/by/4.0/deed.es] que permite el uso, distribución y reproducción en cualquier medio, siempre que la obra original sea debidamente citada de su fuente original 
The association of gastrointestinal parasitism with body weight, haematocrit and the FAMACHA method was determined in Junín sheep (lambs and rams) from the Stud group of SAIS Túpac Amaru Ltda. No. 1, Junín Region, Peru. The study was carried out in two seasons: May (dry) and October (rainy). In total, 109 rams and 98 lambs were sampled in May and 89 rams and 85 lambs in October 2018. There was a negative correlation between the haematocrit and the transformed value of parasite load. All the animals were positive to gastrointestinal parasites, finding a higher parasite load in males in October and in May in females. In May there were more cases of animals resistant to parasitism than in October. The highest frequency of gastrointestinal parasites was Nematodirus (50\%), followed by Trichostrongylus (25\%) and Ostertagia (15\%).

Key words: FAMACHA, haematocrit, gastrointestinal nematodes, body weight, lamb

\section{INTRODUCCIÓN}

La producción ovina en el Perú representa, en gran medida, un soporte económico trascendente para un gran sector de la población rural (Moya y Torres, 2008); especialmente aquellas que habitan en zonas donde el desarrollo de la agricultura presenta limitantes, tanto por condiciones climáticas como altitudinales. La presencia de parásitos gastrointestinales en producciones ovinas suele causar graves pérdidas económicas a los productores cuando se presentan en explotaciones donde algunas prácticas de manejo favorecen la infección por nemátodos gastrointestinales (Lara, 2003), lo que representa una seria limitación para estos sistemas productivos (Vásquez et al., 2001), por una disminución de la productividad. A esto se añade la resistencia a los antiparasitarios convencionalmente utilizados para combatirlas, tales como los benzimidazoles (albendazol), imidazotiazoles (levamisol) o endectocidas (ivermectinas), que es cada vez más intensa y constituye un serio problema por resolver (Van Wyk, 2001; Lara, 2003).
Las parasitosis en general pueden alterar el bienestar animal independientemente del sistema productivo (Herrera et al., 2013), teniendo efectos negativos directos (ganancia de peso, desarrollo corporal, comportamiento reproductivo) e indirectos (subutilización del recurso forrajero y la predisposición a enfermedades) (Soca et al., 2005), además de los costos implicados en los tratamientos del animal, reduciendo la rentabilidad (Márquez, 2003). Esta problemática es importante sobre todo en la crianza de ovinos en la sierra central del país, donde la prevalencia general de parasitismo gastrointestinal por nematodos puede alcanzar valores altos (Puicón et al., 2018).

En este contexto, conocer el nivel de asociación entre los cuadros de nematodiasis gastrointestinal del ganado ovino con la modificación patológica de ciertos parámetros fisiológicos como pérdida de peso, cuadros de anemia y disminución de los valores de hematocrito, resultará importante para entender la dinámica de estas parasitosis con el fin de implementar medidas de control adecuadas. El objetivo del presente estudio, por lo 
tanto, fue determinar el nivel de asociación entre los cuadros de nematodosis gastrointestinal y la variación patológica de parámetros fisiológicos en ovinos de raza Junín.

\section{Materiales y Métodos}

\section{Lugar del Estudio}

El estudio se llevó a cabo en la Unidad de Producción Pachacayo de la SAIS Túpac Amaru Ltda. $\mathrm{N}^{\circ} 1$, ubicada en el distrito de Canchayllo, provincia de Jauja, Región Junín, Perú, entre los 3600 y 4700 msnm. La crianza del ovino Junín es exclusivamente a campo abierto, a base de pastos nativos, sin recurrir a la estabulación ni a la alimentación suplementaria.

Las pruebas diagnósticas de laboratorio se realizaron en los laboratorios de Sanidad Animal y Microbiología de la Facultad de Zootecnia de la Universidad Nacional del Centro del Perú, Huancayo, Región Junín. El trabajo se realizó entre marzo y diciembre de 2018, con dos muestreos de campo en los meses de mayo y octubre.

\section{Población y Muestra}

La población objetivo fue el total de carnerillos y borreguillas (animales destetados), de 4 a 6 meses de edad del lote Stud de la raza Junín. Se trabajó con la totalidad de los animales, considerado como conjunto completo (CEIBAL, 2007). Así, en la primera colección de muestras (mayo) se trabajó con 109 carnerillos y 98 borreguillas y en la segunda (octubre) con 89 carnerillos y 85 borreguillas.

\section{Procedimiento}

Los animales, identificados con aretes numerados, fueron pesados en una balanza tipo reloj: Se tomaron muestras de heces directamente del recto y de sangre de la vena cefálica en tubos vacutainer con EDTA. Ade- más, se registró el nivel de anemia mediante el método FAMACHA ${ }^{\circledR}$, desarrollado en Sudáfrica y que se basa en el tratamiento selectivo de los animales en función de la coloración de la mucosa de la conjuntiva ocular relacionada con los diferentes grados de anemia, para el control de parásitos hematófagos, especialmente Haemonchus spp (van Wyk y Bath, 2002). Las muestras se llevaron al laboratorio en cajas térmicas para su procesamiento y análisis.

El contaje de huevos de los parásitos gastrointestinales (HPG) se realizó por el método McMaster (Hansen y Perry, 1994), con el fin de categorizar los niveles de infestación por animal (Morales y Pino, 2009) de acuerdo con lo indicado en el Cuadro 1. La identificación de las larvas se hizo con el método de Corticelli y Lai modificado (LuckMontero et al., 2018), realizando pooles de muestras de heces para cada nivel de parasitismo, sexo y época. La determinación del hematocrito se hizo mediante la técnica de microhematocrito.

Los animales fueron clasificados por su condición de resistencia al parasitismo según los resultados de la coproscopía cuantitativa (carga parasitaria) y el valor del hematocrito (Morales et al., 2011) (Cuadro 2). No se consideró la lectura de la tarjeta FAMACHA como criterio en esta clasificación, de acuerdo con la propuesta de Morales et al. (2011), debido a que este criterio solo es válido para parásitos gastrointestinales hematófagos como los del género Haemonchus.

Cuadro 1. Niveles de parasitismo gastrointestinal según el número de huevos por gramo de heces (hpg) en ovinos

\begin{tabular}{lcc}
\hline Infestación & hpg & Niveles \\
\hline Baja & $1-50$ & $0-1.7$ \\
Leve & $>50-200$ & $>1.7-2.3$ \\
Moderada & $>200-800$ & $>2.3-2.9$ \\
Alta & $>800$ & $>2.9$ \\
\hline
\end{tabular}


Cuadro 2. Clasificación de ovinos según el valor del hematocrito (Hto) y el nivel de resistencia al parasitismo gastrointestinal

\begin{tabular}{lcc}
\hline Carga parasitaria & $\begin{array}{l}\text { Hto } \\
(\%)\end{array}$ & Nivel \\
\hline Baja y leve & $>38$ & Resistente \\
Moderada y alta & $>38$ & Resiliente \\
Moderada y alta & $\leq 38$ & Sensible \\
\hline
\end{tabular}

\section{Análisis Estadistico}

Se evaluó la normalidad de datos, donde el peso vivo y el hematocrito fueron variables normales, mientras que el hpg fue transformado en base logarítmica decimal para ser normalizada. Para el análisis de los datos se utilizó la correlación de Pearson entre el peso vivo, hematocrito y el hpg en base logarítmica decimal. Para evaluar diferencias entre sexos y estaciones con respecto al peso vivo, hpg en base logarítmica y porcentaje de hematocrito se hizo uso del modelo lineal de efectos fijos. Asimismo, la asociación entre el nivel de parasitismo y resistencia con el sexo y la estación se determinó con la prueba de Chi cuadrado de dependencias, utilizando la prueba de Mantel-Haenszel para probar la asociación condicional de las dos variables binarias (sexo y época) en presencia de una tercera variable categórica (nivel de parasitismo). Todos los análisis se hicieron con el paquete $\mathrm{R}$ v. 3.6.1.

\section{Resultados y Discusión}

\section{Época del Año y Sexo}

No se encontró diferencia significativa entre el peso vivo con las épocas de muestreo (sequía y lluvias) (Cuadro 3). No obstante, el peso promedio de los machos fue significativamente mayor que el de las hembras $(\mathrm{p}<0.05)$, lo cual concuerda con Castellaro et al. (2015) y Bianchi (2007), quienes señalan que la diferencia entre sexos se incrementa con la edad y las mejoras nutricionales.

El menor peso promedio de las borreguillas en la estación de inicio de lluvias (octubre) con relación al inicio de la época de sequía (mayo) se explica por el hecho de que estos lotes tuvieron diferentes zonas de pastoreo por consideraciones de manejo de la empresa, lo que se ve reflejado en un mayor rango de los pesos.

Los valores de hematocrito fueros significativamente mayores en la época de sequía $(41.0 \pm 0.17)$ que en la época de lluvias (39.7 $\pm 0.18 ; p<0.05)$, mientras que fue similar entre sexos. Los valores de hematocrito, sin embargo, se mantuvieron dentro del rango normal del ovino (30-60\%; Andina Premier, 2015). Por otro lado, si se considera el efecto de la altitud, los valores del presente estudio fueron más altos que otros reportados a menor altitud (Morales et al., 2011). En un estudio llevado a cabo en Ayacucho en condiciones de altura, Ayala (2014) obtuvo valores de 43.9 y $36.2 \%$ de hematocrito para machos y hembras, respectivamente, con valores más alto en ovinos de 14- 16 meses en comparación con animales de $8-10$ meses $(38.8 \%)$.

Los coeficientes de correlación de Pearson fueron bajos para peso vivo $\mathrm{y}$ hematocrito (0.031) y para el peso vivo con el hpg en base logarítmica decimal (0.087). La única correlación significativa $(\mathrm{p}<0.05)$ se dio entre el hematocrito y el hpg en base logarítmica decimal (-0.12), lo que indica que a mayor hpg tiende a disminuir el valor del hematocrito en los ovinos del estudio.

La relación entre los niveles FAMACHA y los valores del hematocrito mostraron una significativa relación (Cuadro 4), mientras que los valores transformados de hpg no mostraron diferencias significativas entre los niveles de FAMACHA (Cuadro 4). Esto permite asumir que el uso de FAMACHA no resulta significativo para 
Cuadro 3. Promedios y rangos de peso vivo, hematocrito (Hto) y número de huevos por gramo de heces (hpg) en borreguillas y carnerillos de la SAIS Túpac Amaru durante las épocas de sequía y de lluvias (2018)

\begin{tabular}{lcccccccc}
\hline & \multicolumn{3}{c}{ Sequía (mayo) } & \multicolumn{4}{c}{ Lluvias (octubre) } \\
\cline { 2 - 8 } & \multicolumn{2}{c}{ Machos } & \multicolumn{2}{c}{ Hembras } & \multicolumn{2}{c}{ Machos } & Hembras \\
\hline Peso (kg) & 41.4 & $30-52$ & 42.5 & $34-62$ & 45.7 & $32-55$ & 36.8 & $25-59$ \\
Hto (\%) & 41.0 & $35-47$ & 41.2 & $30-50$ & 39.5 & $34-45$ & 39.9 & $35-45$ \\
FAMACHA & 2.24 & $1-4$ & 2.28 & $1-4$ & 3.01 & $1-4$ & 3.09 & $1-4$ \\
\hline
\end{tabular}

Cuadro 4. Asociación de valores FAMACHA, hematocrito y carga parasitaria en ovinos de la SAIS Túpac Amaru (2018)

\begin{tabular}{cccc}
\hline FAMACHA & Hematocrito (\%) & $\begin{array}{c}\text { Huevos por gramos } \\
\text { de heces (hpg) }\end{array}$ & $\log 10(\mathrm{hpg})$ \\
\hline 1 & $42.47^{\mathrm{d}} \pm 0.53$ & 786.85 & $2.90^{\mathrm{a}} \pm 0.06$ \\
2 & $41.24^{\mathrm{c}} \pm 0.17$ & 670.86 & $2.83^{\mathrm{a}} \pm 0.04$ \\
3 & $39.83^{\mathrm{b}} \pm 0.18$ & 746.43 & $2.87^{\mathrm{a}} \pm 0.03$ \\
4 & $38.79^{\mathrm{a}} \pm 0.30$ & 884.17 & $2.95^{\mathrm{a}} \pm 0.04$ \\
\hline
\end{tabular}

$a, b, c, d$ Letras diferentes dentro de columnas indican diferencia significativa según la prueba de Tukey $(p>0.05)$

Cuadro 5. Asociación del nivel de parasitismo gastrointestinal con el sexo y época del año en ovinos de la SAIS Túpac Amaru (2018)

\begin{tabular}{llcccccc}
\hline \multirow{2}{*}{ Época } & \multirow{2}{*}{ Sexo } & \multicolumn{2}{c}{ Leve } & \multicolumn{2}{c}{ Moderada } & \multicolumn{2}{c}{ Alta } \\
\cline { 2 - 8 } & & $\mathrm{n}$ & $\%$ & $\mathrm{n}$ & $\%$ & $\mathrm{n}$ & $\%$ \\
\hline \multirow{3}{*}{ Sequía } & Macho & 11 & 10.1 & 78 & 71.6 & 20 & 18.3 \\
& Hembra & 2 & 2.1 & 31 & 32.0 & 64 & 66.0 \\
\cline { 2 - 8 } & Subtotal & 13 & 6.0 & 109 & 53.0 & 84 & 41.0 \\
\cline { 2 - 8 } Lluvias & Macho & 0 & 0 & 24 & 27.9 & 62 & 72.1 \\
& Hembra & 2 & 2.6 & 50 & 64.1 & 26 & 33.3 \\
\cline { 2 - 8 } & Subtotal & 2 & 1.0 & 74 & 45.0 & 88 & 54.0 \\
\hline
\end{tabular}


Cuadro 6. Asociación del nivel de resistencia al parasitismo gastrointestinal con el sexo y época del año en ovinos de la SAIS Túpac Amaru (2018)

\begin{tabular}{clcccccc}
\hline \multirow{2}{*}{ Época } & \multirow{2}{*}{ Sexo } & \multicolumn{2}{c}{ Resistente } & \multicolumn{2}{c}{ Resiliente } & \multicolumn{2}{c}{ Sensible } \\
\cline { 2 - 7 } & & $\mathrm{n}$ & $\%$ & $\mathrm{n}$ & $\%$ & $\mathrm{n}$ & $\%$ \\
\hline \multirow{2}{*}{ Sequía } & Macho & 11 & 10.1 & 88 & 80.7 & 10 & 9.2 \\
& Hembra & 2 & 2.1 & 83 & 85.6 & 12 & 2.4 \\
\multirow{3}{*}{ Lluvias } & Macho & 0 & 0 & 60 & 69.8 & 26 & 30.2 \\
& Hembra & 2 & 2.6 & 54 & 69.2 & 22 & 28.2 \\
\hline
\end{tabular}

establecer un nivel de parasitismo (hpg), pero podría ser de utilidad para asociarlo con el estado de anemia (valores de hematocrito) en las condiciones del estudio.

No se encontraron diferencias significativas entre épocas del año en los valores transformados del hpg para carnerillos y borreguillas ( $2.84 \pm 0.02$ en el estiaje; $2.91 \pm$ 0.03 en lluvias). Por otro lado, el valor del hpg fue significativamente menor en machos $(2.82 \pm 0.02)$ que en hembras $(2.93 \pm 0.03)$ $(\mathrm{p}<0.05)$.

\section{Parasitismo Gastrointestinal}

No se encontró asociación significativa entre los niveles de parasitismo gastrointestinal con el sexo; sin embargo, hubo asociación significativa y positiva con la época del año al considerar el total de animales (V. Cramer's $=0.17)($ Cuadro 5).

Todos los animales evaluados estuvieron infectados con parásitos gastrointestinales, ya sea de manera leve, moderada o alta; sin embargo, el estadístico Mantel-Haenzel indica que no hubo asociación significativa entre los niveles de parasitismo con época del año y sexo.

$\mathrm{Al}$ evaluar la totalidad de animales, se encontró diferencias significativas $(\mathrm{p}<0.05)$ entre los grupos de niveles de parasitismo con temporada, para los niveles de parasitismo leve y moderado, pero no se encontraron diferencias en el nivel de parasitismo alto. La cantidad de animales con parasitismo leve disminuye al pasar de la época de sequía a la época de lluvias, mientras que los animales con parasitismo alto, se incrementa ligeramente (Cuadro 5). Según Piscoya (2017), en la sierra del Perú, donde el frio, la lluvia y el calor son muy intensos, en la época de lluvia existe una mayor prevalencia de parásitos intestinales, ya que las lluvias generan un ambiente adecuado para su desarrollo.

\section{Resistencia al Parasitismo Gastroin- testinal}

Los animales fueron clasificados por su condición de resistencia al parasitismo según los resultados de la coproscopía cuantitativa (carga parasitaria) y el valor del hematocrito. En época de sequía se presentaron más casos de animales resistentes al parasitismo que en época de lluvias, al igual que el número de animales resilientes; sin embargo, hubo un incremento de animales sensibles en la estación de lluvias. La cantidad de hembras resilientes disminuyó al pasar de la época de sequía a la época de lluviosa (Cuadro 6); asimismo se encontraron diferencias significativas entre la proporción de animales sensibles con los animales resistentes y resilientes $(\mathrm{p}<0.05)$. 


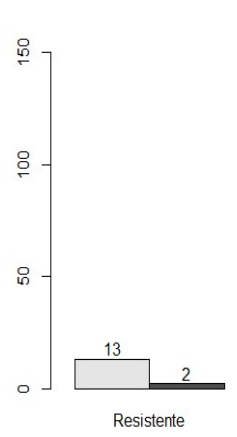

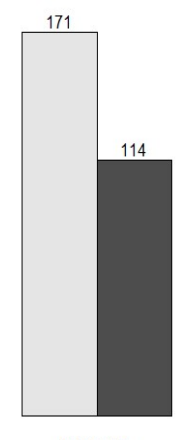

Resiliente
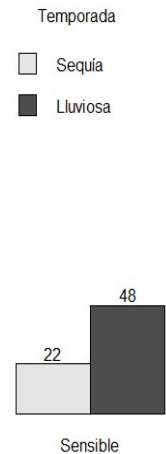

Figura 1. Asociación entre el nivel de resistencia al parasitismo gastrointestinal y la época del año en ovinos de la SAIS Túpac Amaru (2018)

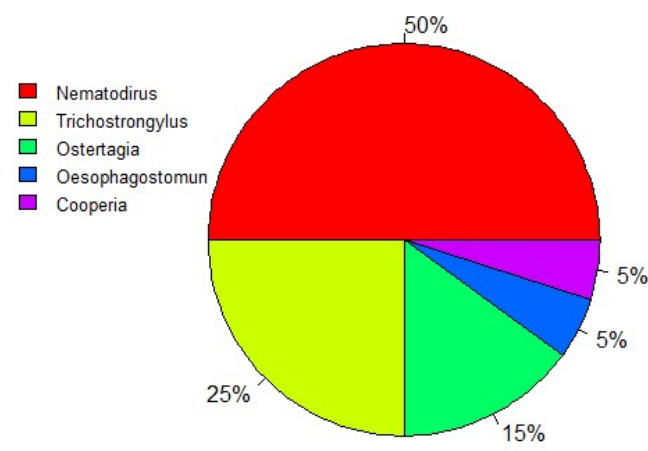

Figura 2. Frecuencia de nematodos gastrointestinales en ovinos de la SAIS Túpac Amaru (2018)

Lope (2016) reportó en 142 ovinos en Tampobata, Perú, que el 40.5, 39.4 y $19.9 \%$ resultaron sensibles, resistentes y resilientes, respectivamente, para la estación de lluvias, mientras que Puicón et al. (2018), evaluando ganado ovino en dos cooperativas de la sierra central, encuentra una gran proporción de animales intermedios (resilientes), seguido de animales sensibles y una pequeña proporción de resistentes, indicando además, que con la pequeña proporción de animales resistentes se podría establecer una población genéticamente mejorada resistente al parasitismo gastrointestinal.
Considerando el total de animales (carnerillos y borreguillas), el grupo de animales sensibles, a diferencia del grupo de animales resistentes y resilientes, tiende a aumentar considerablemente al pasar de la época de sequía a la época de lluvias (Figura 1).

El aumento de animales sensibles al iniciarse el periodo de lluvias puede estar ocasionado por el aumento de la oviposición de los parásitos en estos animales lo que se puede interpretar como consecuencia de la prosecución del desarrollo de las larvas que habrían permanecido hipobióticas durante la época de sequía. Esto se puede considerar como la adaptación de los parásitos para sobrevivir dentro de los animales durante las épocas en que las bajas temperaturas no permiten el desarrollo de las larvas infectantes en la materia fecal (Sievers et al., 2002).

\section{Cultivo de Larvas}

Hubo una mayor presencia de Nematodirus spp (50\%) sobre otras especies de nematodos gastrointestinales (Trichostrongylus spp, 25\%, Ostertagia spp, 15\% y otros), según se muestra en la Figura 2. Los resultados obtenidos son compatibles con lo reportado. En este sentido, Lupaca (2017) en su trabajo en ovinos en el sur del país encontró mayormente Haemonchus spp (23.1\%), Ostertagia spp (22.1\%), Nematodirus spp (15.4\%) y Trichostrongylus spp (14.4\%) Por otro lado, Rojas (2004) cita entre los principales géneros parasitarios para la sierra del Perú a Strongyloides, Toxocara, Trichuris, Bunostomun, Capillaria, Trichostrongylus, Nematodirus, Lamanema, Ostertagia, Cooperia, Haemonchus y Chabertia.

La mayor presencia de Nematodirus spp puede justificarse por la mayor viabilidad de los huevos de esta especie sobre los de tipo estrongílido, ya que durante la época de sequía presentan una gran resistencia frente a la sequedad y bajas de temperatura características de la región altoandina (Leguía, 1991; Leguía y Casas, 1999). 


\section{Conclusiones}

- Todos los ovinos estaban con algún nivel de parasitismo gastrointestinal, especialmente durante la época de sequía.

- Se encontró interacción significativa entre el sexo y la época sobre el peso de los animales $(\mathrm{p}<0.01)$.

- Los valores de hematocrito fueron mayores en época seca.

- Se encontró correlación significativa $(p<0.05)$ entre el hematocrito y el número de huevos por gramo de heces en base logarítmica decimal (-0.12).

- La mayor frecuencia de nematodos fue de Nematodirus (50\%), seguido de Trichostrongylus (25\%), y en menor proporción Ostertagia (15\%), y Oesophagostomun y Cooperia (5\% cada uno).

\section{Literatura Citada}

1. Andina Premier. 2015. App OvinoVet. Aplicación para sistemas de producción de ovino. [Internet]. Disponible en: http:/ /www.andinapremier.com/ovinovet/ category/que-es-el-app/

2. Ayala Y. 2014. La infestación parasitaria gastrointestinal y su influencia sobre los parámetros hematológicos en ovinos jóvenes criollos al pastoreo en la comunidad Urancancha - Víctor Fajardo. Ayacucho-2013. Tesis de Médico Veterinario. Ayacucho: Univ. Nacional de San Cristóbal de Huamanga. 89 p.

3. Bianchi G 2007. Alternativas tecnológicas para la producción de carne ovina de calidad en sistemas pastoriles. Montevideo, Uruguay: Hemisferio Sur. 283 p.

4. Castellaro GG, García FX, Magofke SJC, Marín FG. 2015. Peso vivo y crecimiento de corderos Merino precoz, Suffolk y mestizos en praderas mediterráneas semiáridas de Chile. Chil J Agric Anim Sci 31: 60-69. doi: 10.4067/S071938902016000100007
5. CEIBAL. 2007. Plan Ceibal - Estadística: población y muestra. Montevideo, Uruguay. [Internet]. Disponible en: https:/ /rea.ceibal.edu.uy/elp/estad-stica/ poblacin_y_muestra.html

6. Hansen J, Perry B. 1994. The epidemiology, diagnosis and control of helminth parasites of ruminants. Nairobi, Kenya: International Laboratory for Research on Animal Diseases. 171 p.

7. Herrera L, Ríos L, Zapata R. 2013. Frecuencia de infección por nemátodos gastrointestinales en ovinos y caprinos de cinco municipios de Antioquia. Rev MVZ Córdoba 18: 3851-3860.

8. Lara DM. 2003. Resistencia a los antihelmínticos: origen, desarrollo y control. Cienc Tecnol Agropecu 4: 55-71. doi: 10.21930/rcta.vol4_num1_art:14

9. Leguía P. 1991. Enfermedades parasitarias. En: Avances y perspectivas del conocimiento de los camélidos sudamericanos. Santiago de Chile: FAO. p 325-362.

10. Leguía P, Casas E. 1999. Enfermedades parasitarias y atlas parasitológico de camélidos sudamericanos. Lima, Perú: Ed de Mar. 190 p.

11. Lope R. 2016. Validación de método FAMACHA para diagnóstico de haemonchosis en ovinos de pelo en provincia de Tambopata, Madre de Dios. Tesis de Médico Veterinario y Zootecnista. Puerto Maldonado, Perú: Univ. Nacional Amazónica de Madre de Dios. 30 p.

12. Luck-Montero R, Avendaño-Reyes L, Ail-Catzim C, Cuéllar-Ordaz J, Muñoz-Tenería F, Macías-Cruz $U$. 2018. Actividad ovicida y larvicida de extractos acuosos de Pluchea sericea y Artemisia tridentata en Haemonchus contortus. Ecosistemas y Recur Agropecuarios 5: 149-156. doi: 10.19136/ era.a5n 13.1370

13. Lupaca GJA. 2017. Prevalencia de parásitos gastrointestinales en ovinos de la raza Hampshire Down (Ovis aries) del distrito de Sama, Tacna 2016. Tesis de Médico Veterinario y Zootecnista. Tacna, 
Perú: Univ. Nacional Jorge Basadre Grohmann. $75 \mathrm{p}$.

14. Márquez LD. 2003. Resistencia a los antihelmínticos: origen, desarrollo y control. Corpoica Cienc Tecnol Agropecu 4: 55-71.doi:10.21930/rcta.vol4_num1_art: 14

15. Morales G, Pino AL. 2009. Nematodes parásitos de los rumiantes domésticos en Venezuela: diagnóstico y control. Caraxas, Venezuela: Laboratorio de Diagnóstico Veterinario «Aliani». 143 p.

16. Morales G, Pino AL, Guillen TA, Barrios F, Pinho A. 2011. Clasificación por el método Famacha y su relación con el valor de hematocrito y recuento de h.p.g. de ovinos criados en condiciones de pastoreo. Zootec Trop 28: 545-555.

17. Moya E, Torres J. 2008. Familias alpaqueras enfrentando al cambio climático. Lima. 110 p. [Internet]. Disponible en: https://www.mimp.gob.pe/ webs/mimp/sispod/pdf/186.pdf

18. Piscoya ACL. 2017. Frecuencia de helmintosis intestinal de ovinos en un centro de beneficio de animales de abasto en el distrito de Ate. Tesis de Médico Veterinario. Lima, Perú: Univ. Ricardo Palma. $51 \mathrm{p}$.

19. Puicón NV. 2018. Evaluación de la resistencia natural a nematodos gastrointestinales en alpacas y ovinos en praderas de la puna central del Perú. Tesis de Maestría. Lima, Perú: Univ. Nacional Agraria La Molina. $94 \mathrm{p}$.
20. Puicón V, Chávez J, Gutiérrez G, Sánchez D, More M, Zárate D. 2018. Prevalencia de nematodos gastrointestinales en alpacas y ovinos de dos cooperativas comunales de la región Pasco, Perú. Rev Inv Vet Perú 29: 1440-1448. doi: 10.15381/rivep.v29i4.15189

21. Rojas M. 2004. Nosoparasitosis de los rumiantes domésticos peruanos. $2^{\circ} \mathrm{ed}$. Lima, Perú: Martegraf. 146 p.

22. Sievers G, Jara M, Cárdenas $C$, Núñez J. 2002. Annual study of the of egg and oocyst outputs of gastrointestinal parasites and lungworm larvae in a sheep station of Magallanes, Chile. Arch Med Vet 34: 37-47. doi: 10.4067/S0301732X2002000100004

23. Soca M, Roque E, Soca M. 2005. Epizootiología de los nematodos gastrointestinales de los bovinos jóvenes. Pastos y Forrajes 28: 175-185.

24. van Wyk JA. 2001. Refugia-overlooked as perhaps the most potent factor concerning the development of anthelmintic resistance. Onderstepoort J Vet Res 68: 55-67.

25. van Wyk JA, Bath GF. 2002. The FAMACHA system for managing haemonchosis in sheep and goats by clinically identifying individual animals for treatment. Vet Res 33: 509-529. doi: 10.1051/vetres:2002036

26. Vásquez, Y, Morales G, Pino A, De Moreno L, De Combellas J. 2001. Cronobiología de la emisión de huevos de estróngilos digestivos en ovinos infectados en condiciones naturales. Zootec Trop 19(Supl 1): 279-287. 